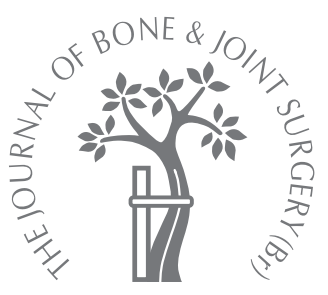

K. Didden,

T. Luyckx,

J. Bellemans,

L. Labey,

B. Innocenti,

H. Vandenneucker

From University

Hospitals UZ,

Pellenberg, Belgium

K. Didden, MD, Resident

Orthopaedic Surgeon

- T. Luyckx, MD, Resident

Orthopaedic Surgeon

In. Bellemans, MD, PhD,

Professor, Orthopaedic

Surgeon, Head of Orthopaedic

Department

W. Vandenneucker, MD,

Assistant Professor

Orthopaedic Surgeon

UZ Leuven, Campus

Pellenberg, Weligerveld 1, 3212

Pellenberg, Belgium.

I. Labey, PhD, Research

Engineer

B. Innocenti, PhD, Research

Enginee

European Centre for Knee

Research

Smith \& Nephew

Technologielaan, 11 bis, 3001

Leuven, Belgium.

Correspondence should be sent to Dr K. D. Didden; e-mail:

karolien.didden@student.kuleu ven.be

(C)2010 British Editorial Society of Bone and Joint Surgery doi:10.1302/0301-620X.92B10. $24221 \$ 2.00$

$J$ Bone Joint Surg [Br] 2010;92-B:1466-70.

Received 10 December 2009;

Accepted 1 June 2010

\title{
Anteroposterior positioning of the tibial component and its effect on the mechanics of patellofemoral contact
}

\begin{abstract}
The biomechanics of the patellofemoral joint can become disturbed during total knee replacement by alterations induced by the position and shape of the different prosthetic components. The role of the patella and femoral trochlea has been well studied. We have examined the effect of anterior or posterior positioning of the tibial component on the mechanisms of patellofemoral contact in total knee replacement. The hypothesis was that placing the tibial component more posteriorly would reduce patellofemoral contact stress while providing a more efficient lever arm during extension of the knee.

We studied five different positions of the tibial component using a six degrees of freedom dynamic knee simulator system based on the Oxford rig, while simulating an active knee squat under physiological loading conditions. The patellofemoral contact force decreased at a mean of $\mathbf{2 . 2} \%$ for every millimetre of posterior translation of the tibial component. Anterior positions of the tibial component were associated with elevation of the patellofemoral joint pressure, which was particularly marked in flexion $>90^{\circ}$.

From our results we believe that more posterior positioning of the tibial component in total knee replacement would be beneficial to the patellofemoral joint.
\end{abstract}

Anterior knee pain remains a common complaint after total knee replacement (TKR). Studies have reported an incidence of anterior knee pain in TKR both with and without patellar resurfacing of about $6.2 \%$ and $25 \%$, respectively. ${ }^{1,2}$ Factors such as the position of the trochlear groove, the rotational position of the femoral and tibial components and the position of the joint line have an important influence on patellofemoral joint biomechanics. ${ }^{3,4}$ However, little is known about the influence of anteroposterior positioning of the tibial tray.

From a biomechanical point of view, a more posterior position of the tibial component would theoretically lead to an improved lever mechanism for extension of the knee, and might reduce patellofemoral contact stress (Fig. 1).

Posterior translation of the tibial component should be comparable to the effect obtained by the Fulkerson and Maquet procedures, 4,5 where anterior displacement of the tibial tuberosity improves the mechanical advantage of the quadriceps and reduces the force in the patellofemoral joint. Hence, these procedures are sometimes used for the treatment of refractory anterior knee pain. ${ }^{3,6,7}$

In TKR where the tibial dimensions fall between two component sizes, the surgeon has the option to select the smaller baseplate and place it some distance away from the anterior margin of the tibia, thereby establishing a more posterior position. The tibia is shifted anteriorly relative to the prosthetic components, providing a 'Maquet'-type effect on the tibial tuberosity and extensor mechanism thereby unloading the patellofemoral joint.

The recent availability of validated dynamic knee simulators, as well as advances in the analysis of contact force and contact area, have made it possible to quantify such an effect. In this study we used a third-generation, custommade, dynamic Oxford knee simulator under physiological loading conditions during a deep knee squat, to study the influence of the position of the tibial component on the patellofemoral contact force, contact area and contact pressure. Our hypothesis was that a more posterior placement of the component would reduce patellofemoral contact stress while providing a more efficient lever arm during extension of the knee.

\section{Materials and Methods}

A dynamic knee simulator based on the Oxford design was customised to simulate and record the dynamic loads during a single squat under conditions of physiological loading, as described below (Fig. 2). The simulator con- 

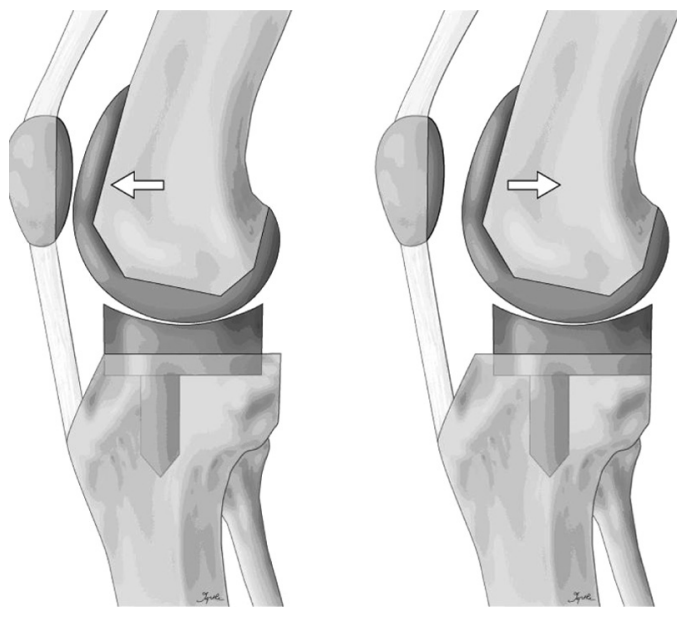

Fig. 1

Diagram demonstrating the effect of changing the anteroposterior position of the tibial component on the patellofemoral joint.
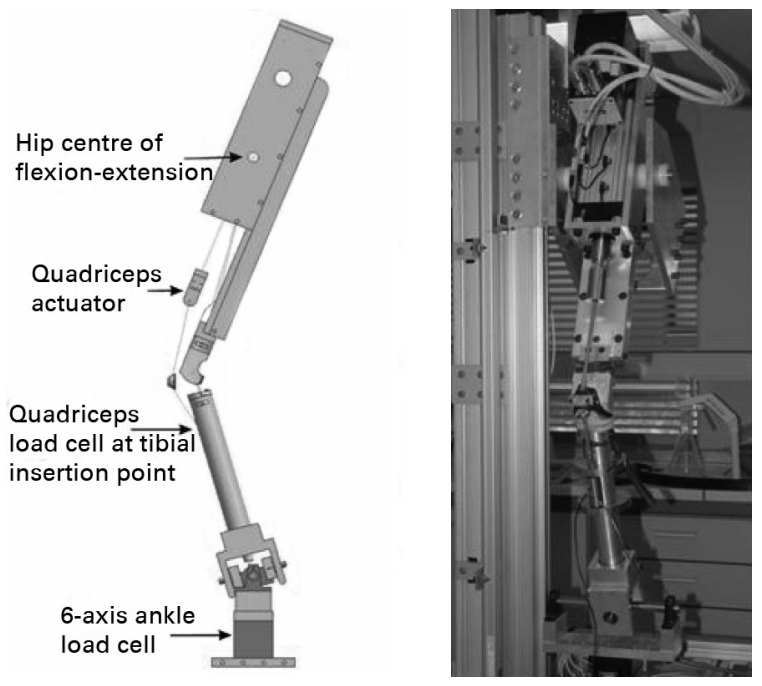

Fig. 2

Diagrammetric representation and photograph of the knee simulator used for the experiments.

sists of two custom-made rigid fixtures, representing an upper and a lower leg-mounted on a frame with a hip and ankle assembly. Two actuators control the movement and loads, one moving the hip and the other pulling the quadriceps. The quadriceps actuator is positioned on the upper leg to anatomically reproduce the moment around the knee. Sensors placed in line with the actuators detect the quadriceps and ankle forces, and the height of the hip relative to the ankle.

The knee simulator is designed with six degrees of freedom: two degrees for movement in the hip joint (vertical translation and rotation around the transverse axis) and four for movement in the ankle joint in order to reproduce normal knee kinematics. ${ }^{8}$ A real-time data acquisition and
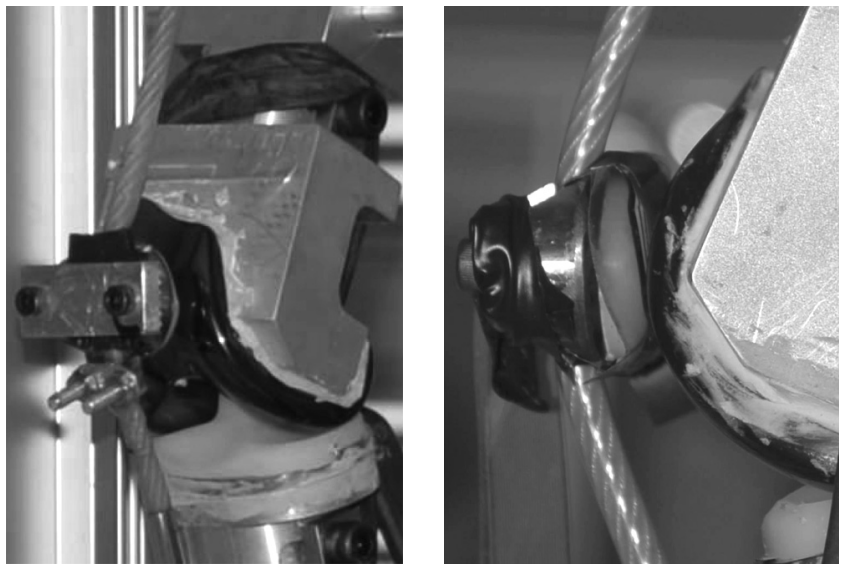

Fig. 3

Retropatellar fixation of the K-scan 4000/9000 pressure sensors (Tekscan, Boston, Massachusetts) to measure the patellofemoral contact area, contact force and contact pressure.

closed feedback system using a LabView module (National Instruments, Austin, Texas) is used to perform a squat at a certain hip velocity, given as a function of time, while simultaneously applying a quadriceps force on the knee to induce a vertical force on the ankle. Error feedback from the six-axis ankle load cell is used to control the quadriceps actuator.

For this experiment a posteriorly-stabilised Genesis II TKR (Smith \& Nephew, Memphis, Tennesee) was attached to the tibia and femur at the simulated knee joint. A size 5 femoral component was used, a size 4 tibial component with a $9 \mathrm{~mm}$ thick insert, and a $32 \mathrm{~mm}$ diameter patellar component to represent a likely clinical combination. A $6.4 \mathrm{~mm}$ diameter stainless steel cable (Sanlo Inc., Michigan City, Indiana) simulated the patellar and quadriceps tendons.

K-Scan 4000/9000 pressure sensors (Tekscan, Boston, Massachusetts) were used to measure contact area, contact force and retropatellar contact pressure. One surface of the sensor was fixed against the articular surface of the patella, using custom wire and adhesive fixtures that did not interfere with the patellofemoral interface (Fig. 3), which was covered entirely by the sensor. The other surface of the sensor was left unattached. A petroleum jelly (Vaseline, Unilever, United Kingdom) was used as a lubricant and applied at the patellofemoral interface between the sensor and the femoral component to reduce shear forces.

The patellar component was attached to the stainless steel cable to recreate a normal Blackburne-Peel Index ${ }^{9,10}$ of 0.84 . The tibial component was tested in five positions in the anteroposterior plane with $+6,+3,0,-3$ and $-6 \mathrm{~mm}$ translation of the centre of the tibial tray relative to the centre of the anteroposterior axis of the tibial plateau. Load and movement curves used for this experiment simulated a single knee squat from $30^{\circ}$ to $120^{\circ}$ and back to $30^{\circ}$ at a 


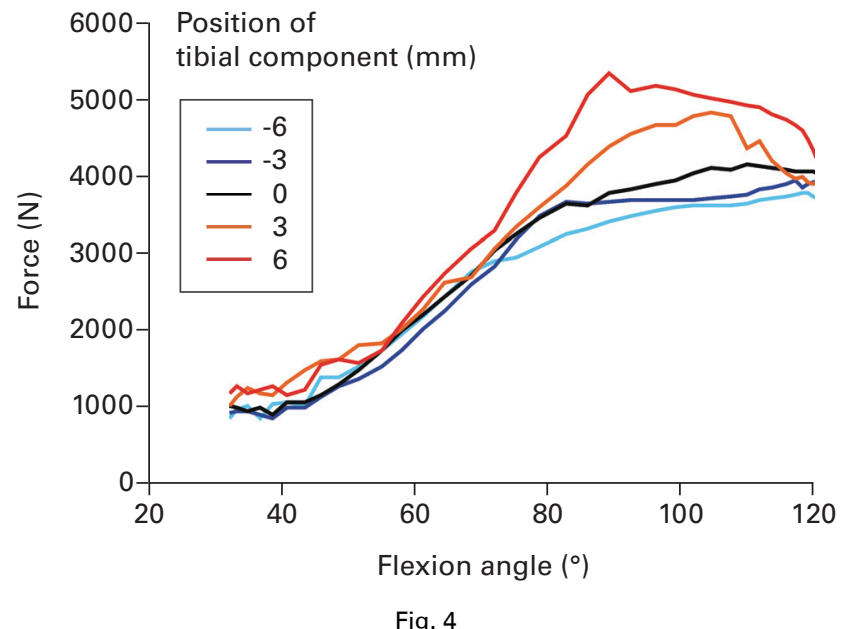

Experimentally determined mean patellofemoral contact force as a function of flexion angle for the different positions of the tibial component.

constant flexion rate over a 20 second interval and with a target ankle load maintained at a constant $267 \mathrm{~N},(\mathrm{~F}=\mathrm{M} \times \mathrm{g}$, $\mathrm{g}=9.81)$ during two-legged squatting, where each ankle would carry half an approximated body weight of $60 \mathrm{~kg}$. This representation of a single knee squat was repeated three times for each position of the tibial component.

Statistical analysis. Pearson's correlation coefficients were used to assess the association between the maximum patellofemoral contact force, contact area and contact pressure and the position of the tibial component. Statistical analyses were performed with SPSS v. 16.0 (SPSS Inc., Chicago, Illinois) with a level of significance set at $\mathrm{p}<0.01$.

\section{Results}

Patellofemoral contact force. For all positions of the tibial component, the patellofemoral contact force increased with increasing knee flexion. It reached a maximum, followed by a reduction, which coincided with the quadriceps tendon contacting the femoral trochlear groove (Fig. 4). This contact occurred at lower angles of flexion for the more anterior positions of the baseplate than with the neutral (zero) position. For the posterior positions, the patellofemoral contact force did not demonstrate a clear peak and the load shearing effect was less obvious, because the quadriceps tendon-femoral contact occurred at flexion angles beyond the range which we tested.

Posterior positions were associated with lower patellofemoral contact forces during the entire squat, despite the fact that the same target load of the ankle was achieved for all tests. At $90^{\circ}$ flexion, the mean contact force was $1945 \mathrm{~N}$ $(36 \%)$, less for the most posterior position $(3396 \mathrm{~N})$ than for the most anterior $(5341 \mathrm{~N})$.

Plotting the mean maximum patellofemoral contact forces as a function of the position of the tibial component revealed a linear trend, with an increase in maximum contact force with the anterior positions (Fig. 5). The relative

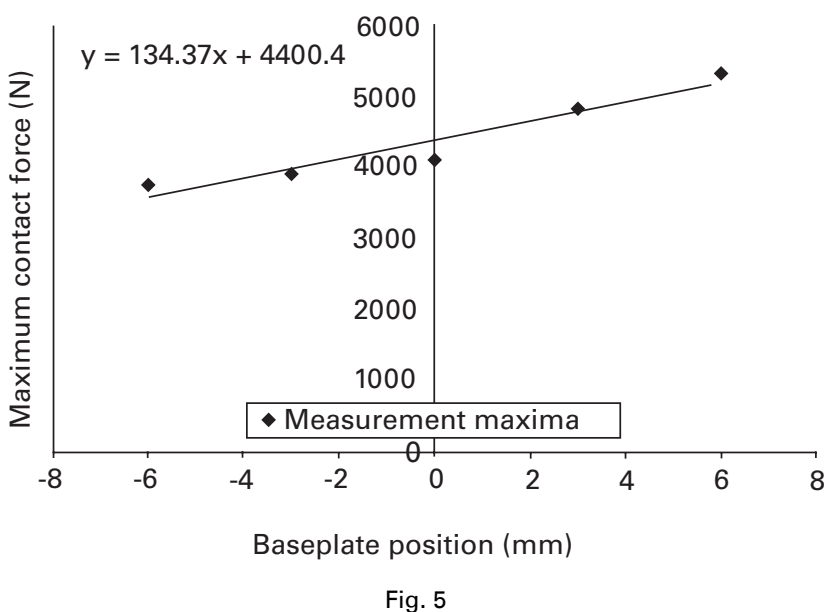

Measured mean maximum contact forces for the different tibial component positions.

change in contact force was defined as the difference between the contact force measured at the different positions and at the neutral placement, divided by the contact force at the latter site. As such, the patellofemoral contact force had a mean reduction of $2.2 \%$ for every millimetre of posterior translation of the tibial component.

Patellofemoral contact area. For all positions of the tibial component the patellofemoral contact area gradually increased with increasing knee flexion, reaching a maximum at an angle ranging from $70^{\circ}$ to $95^{\circ}$ (Fig. 6). The maximum contact area occurred at the angle of flexion where there was a transition of the patella from the femoral trochlear groove on to the two femoral condyles. When the tibial component was positioned in neutral the contact area reached its maximum at $90^{\circ}$ of knee flexion.

Anterior positions of the tibial component were associated with larger contact areas. The mean difference between the maximum contact area of the most anterior $\left(191 \mathrm{~mm}^{2}\right)$ and the most posterior $\left(134 \mathrm{~mm}^{2}\right)$ position of the baseplate was $57 \mathrm{~mm}^{2}(30 \%)$.

Patellofemoral contact pressure. During the entire squat, the mean patellofemoral contact pressure was higher for tibial components with more anterior positions (Fig. 7). The relationship between increasing contact pressure and a more anterior tibial position, however, was not linear. The most posterior position (6 $\mathrm{mm}$ posterior) had a higher mean contact pressure $(26.2 \mathrm{MPa})$ than the neutral and the $3 \mathrm{~mm}$ posterior positions. The lowest mean contact pressure over the entire squat $(24.6 \mathrm{MPa})$ was at the $3 \mathrm{~mm}$ posterior position.

In initial flexion from $30^{\circ}$ to $80^{\circ}$ the contact pressure of all positions of the tibial component fluctuated between $19 \mathrm{MPa}$ and $29 \mathrm{MPa}$. At greater flexion $\left(90^{\circ}\right.$ to $\left.120^{\circ}\right)$, at the stage where the patella leaves the patellofemoral groove and reaches the femoral condyles, a decrease in the contact area and increase in contact pressure was noted for all positions. 


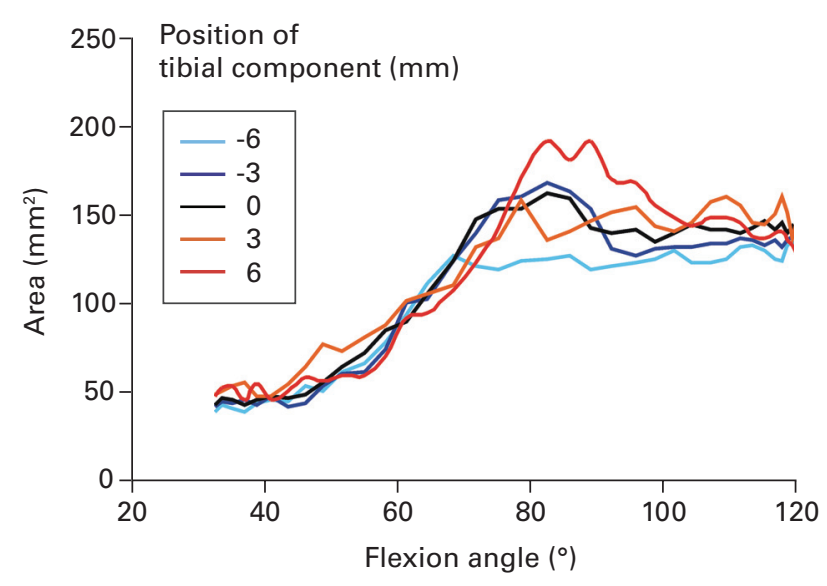

Fig. 6

Experimentally determined mean patellofemoral contact area for the different tibial component positions as a function of the flexion angle.

The mean contact pressure in deeper flexion varied between $28 \mathrm{MPa}$ and $34 \mathrm{MPa}$. The maximum contact pressure for the neutral and posterior positions was always less than $30 \mathrm{MPa}$, but reached $34 \mathrm{MPa}$ for the most anterior position.

Patellofemoral contact forces during the extension phase of the squat were approximately $10 \%$ higher than during the flexion phase, but qualitatively, the effect of the position of the tibial component on the contact force, contact area and contact pressure as a function of the flexion angle was the same for both the flexion and the extension cycles.

\section{Discussion}

Our results demonstrate that posterior positioning of the tibial component leads to lower patellofemoral contact forces when performing a simulated knee squat. These lower forces can be explained biomechanically by the more posterior position of the centre of rotation of the knee, which leads to an increased moment arm of the patellar tendon and hence to a more efficient use of the extensor mechanism (Fig. 1). ${ }^{4,11}$

Anterior displacement of the tibial tubercle, as used in the Maquet and Fulkerson procedures, ${ }^{4,5}$ is based on the same principle. The measurements of Shirazi-Adl and Mesfar ${ }^{5}$ demonstrate that at full extension elevation of the tubercle by $1.25 \mathrm{~cm}$ results in a $1 \mathrm{~cm}$ posterior tibial shift, which diminishes the patellofemoral joint reaction force, an effect that is less obvious with increasing knee flexion.

Maquet $^{12}$ identified that $2 \mathrm{~cm}$ of anterior displacement was required to reduce the patellofemoral contact force by $50 \%$ during the gait cycle. Our data demonstrated a $2.2 \%$ reduction in the mean patellofemoral contact force with each millimetre of posterior displacement, which is of a similar order to Maquet's findings. This is broadly consistent with the data of Singerman et al, ${ }^{13}$ who noted a $17 \%$ reduction in patellofemoral contact force for a $1 \mathrm{~cm}$ advancement of the tibial tubercle. Our data therefore sug-

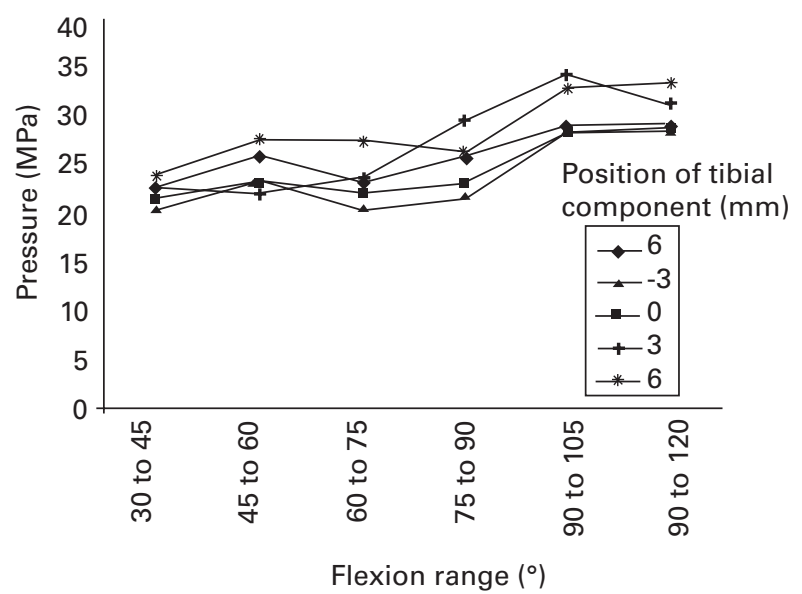

Fig. 7

Mean contact pressure for the different tibial component positions as a function of flexion.

gest that a more posterior position of the tibial component is beneficial, as it unloads the patellofemoral joint.

Surgeons generally determine the position of the tibial component based upon the best fit achievable on the resected surface. Obtaining cortical support in order to provide solid seating of the component while avoiding overhang is usually the main criterion applied in choosing its size as well as its placement. Bloebaum et $\mathrm{al}^{14}$ showed that the tibial cortical bone is significantly thicker in the posterior and medial areas of the tibial plateau. Based on their data, the most posteromedial position of the tibial tray provides the best bony support for fixation.

Where the surgeon has the choice between a more anterior or posterior position for the tibial component, the more posterior is advisable because of stronger bone support and the reduction in the patellofemoral forces.

Where an optimum fit is available, the component cannot be shifted without creating overhang. However, when the dimensions of the tibia fall in between two sizes of component, the surgeon has the option to choose the smaller baseplate, and may shift the position away from the anterior margin of the tibia, thereby establishing a more posterior position.

Our study demonstrated lower patellofemoral contact forces with more posterior positions of the tibial component, despite the smaller contact areas that occurred. Nevertheless, the resulting patellofemoral stress during the squat was less. The patellofemoral contact area for all positions reached a maximum at around $90^{\circ}$ of flexion and decreased thereafter as the patella made the transition from one central contact area on the femoral trochlear to two separate, smaller areas on the two femoral condyles, causing an overall reduction in the patellofemoral contact area.

The patellofemoral contact pressure gradually increased with flexion for all positions of the tibial component (Fig. 7). In deeper flexion the contact pressure ranged 
between $28 \mathrm{MPa}$ and $34 \mathrm{MPa}$, which exceeds the tensile yield strength of ultra high molecular weight polyethylene, which has been reported to be $25.6 \mathrm{MPa}$ (SD 3.3). ${ }^{15}$ Contact between the metal and the polyethylene components in a total joint replacement results in complex stress distributions on the surface and within the polyethylene. In itself, the compressive stress that we measured is not sufficient to explain damage to the polyethylene, as observed for example in retrieved components. These observations must be explained by the range of cyclic stresses that the polyethylene experiences during a load cycle. It has been shown that if this range exceeds $23 \mathrm{MPa}$, UHMWPE fails in fewer than $10^{6}$ cycles. ${ }^{16,17}$ Patellofemoral contact pressures exceeding this yield stress will cause damage to the polyethylene and jeopardise the longevity of the implant. For the more anterior positions, the patellofemoral contact pressure was continuously greater than $30 \mathrm{MPa}$ as soon as flexion exceeded $90^{\circ}$.

The anteroposterior positioning of the tibial component influences patellofemoral contact mechanics in TKR, with a more anterior position leading to increased stresses on the patellofemoral joint. When the surgeon has the option to position the tibial component more posteriorly, this would be beneficial to the joint.

\section{Listen live}

Listen to the abstract of this article at www.jbjs.org.uk

No benefits in any form have been received or will be received from a commercial party related directly or indirectly to the subject of this article.

\section{References}

1. Garneti N, Mahadeva D, Khalil A, McLaren CA. Patellar resurfacing versus no resurfacing in Scorpio total knee arthroplasty. J Knee Surg 2008;21:97-100.

2. Waters TS, Bentley G. Patellar resurfacing in total knee arthroplasty: a prospective, randomized study. J Bone Joint Surg [Am]2003;85-A:212-17.

3. Hungerford DJ, Barry M. Biomechanics of the patellofemoral joint. Clin Orthop 1979;144:9-15.

4. Maquet P. Advancement of the tibial tuberosity. Clin Orthop 1976;115:225-30.

5. Shirazi-Adl A, Mesfar W. Effect of tibial tubercle elevation on biomechanics of the entire knee joint under muscle loads. Clin Biomech 2007;22:344-51.

6. Bellemans J. Biomechanics of anterior knee pain. Knee 2003;10:123-6.

7. Hehne HJ. Biomechanics of the patellofemoral joint and its clinical relevance. Clin Orthop 1990;258:73-85.

8. Zavatsky AB. A kinematic-freedom analysis of a flexed-knee stance testing rig. J Biomech 1997;20:277-80.

9. Blackburne JS, Peel TE. A new method of measuring patellar height. J Bone Joint Surg [Br] 1977;59-B:241-2.

10. Seil R, Muller B, Georg T, Kohn D, Rupp S. Reliability and interobserver variability in radiological patellar height ratios. Knee Surg Sports Traumatol Arthrosc 2000;8:231-6.

11. D'Lima D, Poole D, Chadha H, et al. Quadriceps moment arm and quadriceps forces after total knee arthroplasty. Clin Orthop 2001;392:213-20

12. Maquet P. Mechanics and osteoarthritis of the patellofemoral joint. Clin Orthop 1979;144:70-3.

13. Singerman R, Heiple K, Davy DT, Goldberg V. Effect of tibial component position on patellar strain following total knee arthroplasty. J Arthroplasty 1995;10:651-6.

14. Bloebaum DR, Bachus KN, Mitchell W, Hoffman G, Hofmann AA. Analysis of the bone surface area in resected tibia: implications in tibial component subsidence and fixation. Clin Orthop 1994;309:2-10.

15. Lewis G. Properties of crosslinked ultra-high-moleclar-weight polyethylene. Biomaterials 2001;22:371-401.

16. Weightman B, Light D. A comparison of RCH 1000 and Hi-Fax 1900 ultra-high molecular weight polyethylenes. Biomaterials 1985;6:177-83.

17. Bartel DL, Bicknell VL, Wright TM. The effect of conformity, thickness, and material on stresses in ultra-high molecular weight components for total joint replacement. $J$ Bone Joint Surg [Am] 1986;68-A:1041-51. 